\title{
Nonadiabatic combustion waves in a two-step competitive exothermic-reaction model
}

\author{
Z. Huang ${ }^{1} \quad$ H. S. Sidhu ${ }^{2} \quad$ I. N. Towers ${ }^{3}$ \\ Z. Jovanoski ${ }^{4}$ V. V. Gubernov ${ }^{5}$
}

(Received 23 December 2015; revised 24 May 2016)

\begin{abstract}
We consider travelling front solutions of a one-dimensional reactiondiffusion system corresponding to two-stage competitively exothermic reactions. We suppose all reactions occurring during the combustion may be lumped together as two different paths. Both exothermic reactions compete for the same reactant. Properties of travelling wave fronts, particularly flame speed, are determined numerically by solving the governing partial differential equations. The flame speed is analysed for different values of the heat loss parameter. It is demonstrated that, as the heat loss coefficient increases, the flame speed decays gradually until the front ceases to exist due to insufficient energy being available to sustain the flame front. Earlier studies for the adiabatic case showed
\end{abstract}

http://journal austms.org.au/ojs/index.php/ANZIAMJ/article/view/10391 gives this article, (c) Austral. Mathematical Soc. 2016. Published June 3, 2016, as part of the Proceedings of the 12th Biennial Engineering Mathematics and Applications Conference. ISSN 1446-8735. (Print two pages per sheet of paper.) Copies of this article must not be made otherwise available on the internet; instead link directly to this URL for this article. 
the existence of bi-stability (fast and slow waves co-exist for the same parameter values). We study how heat loss affects the size of the bi-stable region. Furthermore, we investigate how the extinction limit depends on the heat loss parameter as well as the parameter representing the ratio of the activation energy to the heat release of the second reaction. Numerical solutions show that there is no travelling front when these parameters are above threshold values. The dependence of flame speed on the temperature profile is also investigated. The bistability phenomenon is demonstrated by perturbing the temperature profile.

\section{Contents}

1 Introduction

2 Governing equations

3 Numerical solutions

4 Conclusion

References

\section{Introduction}

The study of combustion is significantly important for a wide range of applications, particularly in energy production and the synthesis of advanced materials. Recent experiments by Choi et al. [1] showed that self-propagating thermopower waves in carbon nanotubes can convert chemical energy directly to electrical energy with high specific power. Furthermore, solid flame combustion and self-propagating high-temperature synthesis have been developed 
intensively and used widely in materials technology [2]. Hence, there is tremendous value in investigating mathematical models that simulate combustion processes. Of particular interest are reaction-diffusion systems describing combustion processes that admit travelling wave solutions, also referred to as combustion waves. Such systems are characterized by highly nonlinear dependence of the reaction rate on temperature.

A general combustion process may involve hundreds or even thousands of concurrent or consecutive reactive steps, some of which are exothermic and some endothermic. In order to mathematically analyse the overall combustion process, it is common to use the reduced-kinetic approach to reduce a large set of elementary reactions into a smaller set of 'lumped' global reactions. For example, the detailed schemes of hydrogen and methane oxidation involving dozens of intermediate reactions were successfully reduced to several steps [3, 4, 5, 6, 7]. Sánchez et al. [5] investigated lean premixed one-dimensional methane-air flames using both the detailed and reduced kinetic mechanisms. The numerical results showed that the reduced-kinetic model accurately predicted the main characteristics of the lean premixed combustion wave such as flame front speed and flame structures including the profiles of the temperature and reactants. A similar type of analysis was carried out by Sánchez et al. [6] in the case of ignition of hydrogen-air diffusion flames. The numerical analysis of flame behaviour comparing the full conservation equations with detailed chemistry and the reduced three-step kinetic scheme showed excellent agreement. Gubernov et al. [7] investigated the stability of premixed rich hydrogen-air flames with a two-step reduced kinetic mechanism and demonstrated that the two-step models accurately predict the properties of the combustion waves, including, for first time, the flame stability.

Lumped models are obtained in some cases through a reduction of the detailed kinetic mechanism. The simplest model is the single irreversible exothermic reaction model. However, in some situations, it is necessary to take into consideration several reaction steps, particularly two-stage reactions. If both reactions consume the same reactant, then the reactions are referred to as competitive reactions, but if each reaction independently feeds on different 
reactive material, then they are referred to as parallel reactions [8]. Parallel reactions are only thermally coupled, whereas in the competitive case, the reactions are chemically coupled as well as thermally coupled. In a two-stage sequential scheme $(\mathrm{A} \rightarrow \mathrm{B} \rightarrow \mathrm{C})$, the reactions are both chemically and thermally coupled.

In this article we only focus on the investigation of premixed combustion waves in a model with two-step competing exothermic reactions in a premixed one-dimensional reactive medium. Such reactions have direct relevance to the combustion of $\mathrm{ZrCH}_{2}$ [9]. Sidhu et al. [10] investigated flame propagation in a model with two-stage competitive exothermic reactions and demonstrated the existence of regions of bi-stability under adiabatic conditions. Distinct stable travelling wave solutions were found numerically by solving the governing partial differential equations (PDEs) when varying the ignition conditions. Towers et al. [11] affirmed the existence of multiplicity in combustion wave behaviour based on an ordinary differential equation (ODE) formulation, and found a third unstable branch joining the two stable branches in some region of parameter space. A hysteresis type of behaviour was also demonstrated by varying the activation energy of one of the reactions. The analysis reported here extends the previous studies to take heat loss into consideration. The effects of heat loss on the behaviour of travelling waves and on the regions of bi-stability are examined.

\section{Governing equations}

We consider a model in one dimension that involves two independently irreversible exothermic reactions:

$$
A \stackrel{k_{1}(T)}{\longrightarrow} B+Q_{1}, \quad A \stackrel{k_{2}(T)}{\longrightarrow} C+Q_{2},
$$

where $A$ represents the reactant; $B$ and $C$ are physically and chemically inert products which during the whole combustion process do not change their 
physical properties, such as density, or heat capacity, nor the diffusivity of the system ; $Q_{1}$ and $Q_{2}$ describe the heat released by the first and second reactions, respectively. The two reaction rates $k_{1}(T)$ and $k_{2}(T)$ are characterised by strong nonlinear dependence on the reaction temperature T. Following Forbes and Derrick [12], the Arrhenius kinetics take the form

$$
k_{i}(T)= \begin{cases}0, & T<T_{i}, \\ A_{i} e^{-E_{i} / R T}, & T>T_{i}, \quad i=1,2,\end{cases}
$$

where $A_{i}$ is the pre-exponential reaction factor and $E_{i}$ is the activation energy for reaction $i=1,2$. The universal gas constant is represented by $R$. The critical temperatures $T_{1}$ and $T_{2}$ are where the first and second reactions occur respectively. For simplicity, we set $T_{1}=T_{2}=T_{a}$ where $T_{a}$ is the ambient temperature.

No reaction occurs when the fuel temperature is below the ignition temperature. Thus, following Huang et al. [13], when $T \geqslant T_{a}$, the nonadiabatic governing partial differential equations (PDEs) derived from the conservation of energy and fuel mass are

$$
\begin{aligned}
\rho c_{p} \frac{\partial T}{\partial t} & =k \frac{\partial^{2} T}{\partial x^{2}}+\rho Q_{1} Y k_{1}(T)+\rho Q_{2} Y k_{2}(T)-\frac{h S}{V}\left(T-T_{a}\right), \\
\frac{\partial Y}{\partial t} & =D \frac{\partial^{2} Y}{\partial x^{2}}-Y k_{1}(T)-Y k_{2}(T) .
\end{aligned}
$$

Here $Y$ represents the fuel mass fraction, $\rho$ is the fuel density, $c_{p}$ is the specific heat of the fuel, $k$ is the thermal conductivity of the fuel, $D$ represents the molecular diffusivity of the fuel, $h$ is the heat transfer coefficient from fuel to the ambient surroundings, S/V represents the surface area to volume ratio of the fuel configuration. The last term in (2) represents Newtonian cooling.

With the introduction of the dimensionless variables

$$
\tau=\left(\frac{Q_{2} A_{2} R}{c_{p} E_{2}}\right) t, \quad \xi=\left(\sqrt{\frac{\rho Q_{2} A_{2} R}{k E_{2}}}\right) x, \quad u=\left(\frac{R}{E_{2}}\right) T, \quad v=Y,
$$


the governing PDEs (2) and (3) are

$$
\begin{aligned}
& \frac{\partial u}{\partial \tau}=\frac{\partial^{2} u}{\partial \xi^{2}}+v e^{-1 / u}+q r v e^{-f / u}-l\left(u-u_{a}\right), \\
& \frac{\partial v}{\partial \tau}=\frac{1}{L e} \frac{\partial^{2} v}{\partial \xi^{2}}-\beta v e^{-1 / u}-r \beta v e^{-f / u}
\end{aligned}
$$

where

$$
\begin{aligned}
& u_{a}=\frac{R T_{a}}{E_{2}}, \quad q=\frac{Q_{1}}{Q_{2}}, \quad f=\frac{E_{1}}{E_{2}}, \quad r=\frac{A_{1}}{A_{2}}, \\
& L e=\frac{k}{\rho c_{p} D}, \quad \beta=\frac{c_{p} E_{2}}{R Q_{2}}, \quad l=\frac{h S E_{2}}{V R \rho Q_{2} A_{2}} .
\end{aligned}
$$

The dimensionless temperature and mass fraction are denoted by $u$ and $v$; $\xi$ and $\tau$ represent non-dimensional space and time coordinates, respectively. The new parameters $q, r$ and $f$ are the ratios of enthalpies, the pre-exponential factors and activation energies, respectively. The parameter $\beta$ is the ratio of activation energy to heat released by the second reaction. The parameter $l$ represents the volumetric heat loss coefficient. The Lewis number Le is the ratio of thermal conductivity to mass diffusivity, varying from around unity for gaseous fuels to 'infinite' for solid fuels [14].

The governing PDEs (5) and (6) are subject to the boundary conditions

$$
\begin{array}{ll}
u=u_{a}, & v=1, \quad \text { for } \xi \rightarrow \infty, \\
u=u_{a}, & \frac{\partial v}{\partial \xi}=0, \quad \text { for } \xi \rightarrow-\infty .
\end{array}
$$

The right boundary condition $(\xi \rightarrow \infty)$ is a 'cold' and 'unburnt' state (the dimensionless ambient temperature is denoted as $\boldsymbol{u}_{\mathrm{a}}$ and the fuel concentration equals to one). However, on the left boundary $(\xi \rightarrow-\infty)$, the fuel is losing heat and is cooling down to the ambient temperature and the fuel concentration cannot be specified. No reaction occurs at this steady state and therefore the derivative of $v$ with respect to $\xi$ is set to zero. The main 
difference between the adiabatic and the nonadiabatic cases lies in the left boundary conditions. In the adiabatic case $u(-\infty)=\mathfrak{u}_{\mathrm{b}}$ and $v(-\infty)=0$, where $\mathfrak{u}_{\mathfrak{b}}$ is the peak value of the temperature profile since there is no heat transfer from the reactions to the surroundings and the fuel is consumed completely. In contrast, for the nonadiabatic case, there is always some leftover of fuel behind the front, and the temperature of the fuel cools down to the ambient value.

\section{Numerical solutions}

Travelling wave solutions are found numerically by solving the governing PDEs (5) and (6) together with the boundary conditions (7) and (8) using FlexPDE $^{\mathrm{TM}}[15]$. FlexPDE ${ }^{\mathrm{TM}}$ is a commercial finite-element package used for obtaining both steady-state and time-dependent numerical solutions of PDEs. FlexPDE ${ }^{\mathrm{TM}}$ is adaptive in nature. In other words, whenever the prescribed error tolerance is reached or exceeded, the mesh size is refined and solutions are recalculated until the estimated errors are within the set tolerance level. In this article the error tolerance limit is set to 0.001 . The solutions obtained using FlexPDE ${ }^{\mathrm{TM}}$ are validated independently by using the method of lines (MOL) [16]. MOL is a standard tool for solving PDEs, which discretises space to reduce an initial boundary value problem to a system of ODEs in time. Then a variable time-step approach with time local error control is employed to solve the system of ODEs.

Since this is a preliminary investigation of the combustion model with heat loss, we fix $L e=2, f=3, q=5$ and $r=25$ for the remainder of the article. The activation energy for the combustion of Zirconium is around $10^{6} \mathrm{~J} \mathrm{~mol}^{-1}$. Therefore, it is reasonable to set the value of $\mathfrak{u}_{a}$ to $10^{-4}$. For the numerical integration of the governing PDEs, the initial temperature profile is a Gaussian function [10]

$$
u=A e^{\left(-0.01 \xi^{2}\right)}+u_{a}
$$


Figure 1: The flame speed versus $\beta$ for four different values of the heat loss coefficient $l$ with $A \geqslant 0.2$. Solutions for $l=0, l=10^{-4}$ and $l=5 \times 10^{-4}$ are respectively represented by the red solid line, green dashed line and blue ring, whereas the magenta triangle corresponds to the solution for $l=10^{-3}$. The solutions representing the unstable branch for the adiabatic case are indicated by the red dashed line obtained via the ODE formulation.

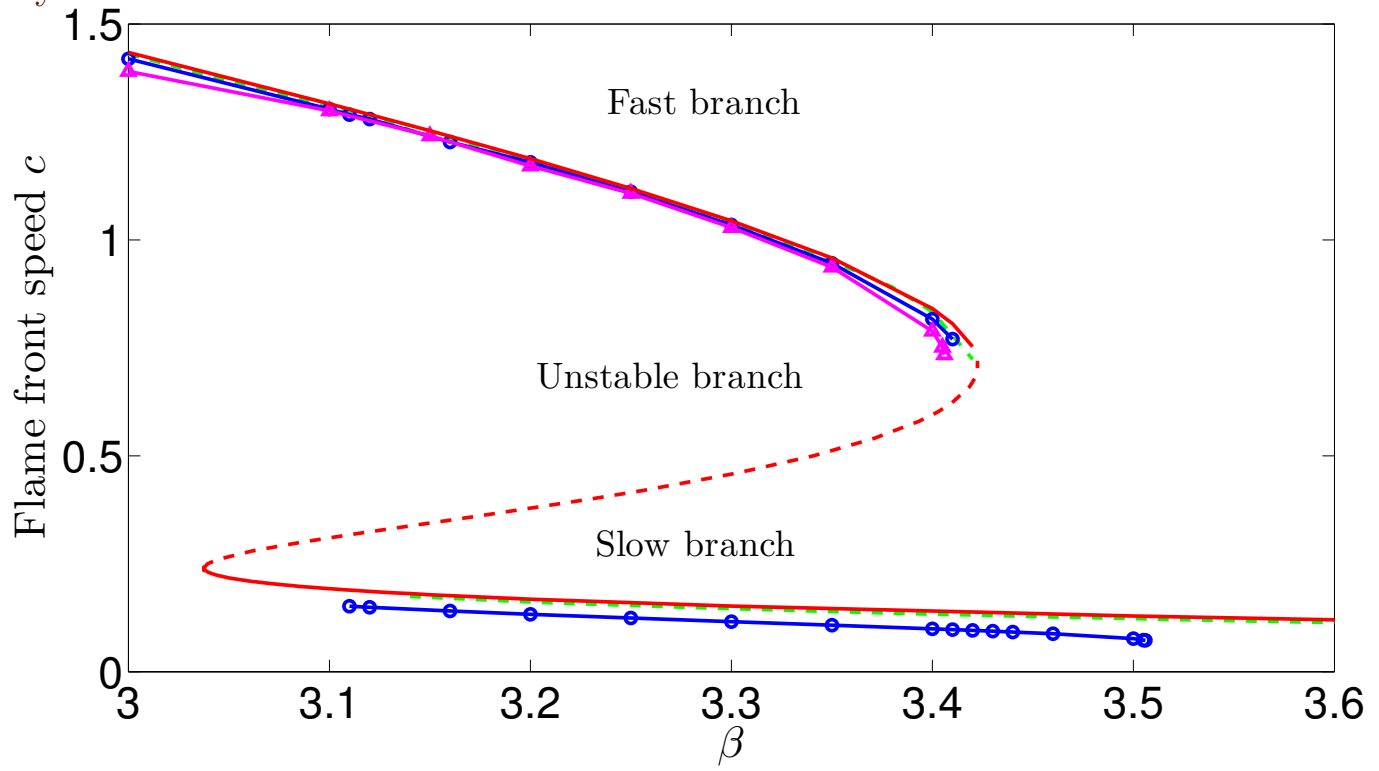

imitating an ignition spark. The amplitude of the Gaussian $A$ is a key parameter in regions where bi-stability exists as different stable solutions are obtained by choosing different initial conditions. The one-dimensional spatial domain and the integration time were set to $0<\xi<10000$ and $0<\tau<$ $5 \times 10^{6}$, respectively, so that a stable propagating front is established.

Figure 1 illustrates the dependence of the flame speed upon the parameter $\beta$ for four different values of the heat loss coefficient $l$. The flame speed decays monotonically as $\beta$ increases before the travelling waves cease to exist. This behaviour is observed in other combustion models such as single-step 
combustion models [17]. Also, the flame speed decreases as the heat loss coefficient rises, since increasing the heat loss results in less energy being available to sustain the propagation of the flame fronts. Figure 1 shows two disjointed branches: the lower branch is associated with flame fronts with slow speeds, the so-called 'slow branch', and the upper branch is associated with flame fronts with fast speeds, the so-called 'fast branch'. By solving the associated ODEs, Towers et al. [11] found that there is a third branch connecting these two branches (the unstable branch for the adiabatic case is indicated by the red dashed line in Figure 1). We focus on the stable solutions since these solutions are of practical interest as they can be observed experimentally.

The regions of bi-stability are seen in Figure 1. When the value of the heat loss coefficient is sufficiently small, namely, $l=10^{-4}$, the region of bi-stability is almost identical with that for $l=0$ (although they have distinctive temperature and fuel profiles). However, when the heat loss coefficient is sufficiently large, namely $l=10^{-3}$, there exists only one unique combustion wave solution branch (magenta triangle in Figure 1) - the slow branch disappears. For the case with $l=0$ and $l=10^{-4}$, the bi-stable region exists for $3.14 \leqslant \beta \leqslant 3.42$, whereas for the case with $l=5 \times 10^{-4}$ bi-stability exists for $3.11 \leqslant \beta \leqslant 3.41$. Hence, the heat loss has an influence on the existence and the size of regions of bi-stability.

As mentioned after equation (9), the amplitude of the Gaussian $A$ is an important parameter. By varying the value of $A$ within the region of bistability the solutions on the fast branch can switch to the slow branch and vice versa. The fast and slow combustion wave profiles shown in Figure 2 are obtained by setting $A=0.5$ and $A=0.2$, respectively. The temperature and fuel concentration profiles of the two solutions have the same qualitative behaviour. However, the flame speeds are quite different - the flame speed for the fast branch is approximately 1.034 , which is much larger than 0.116 for the slow branch. Another distinction is that the peak value of the dimensionless temperature for the fast branch (approximately 1 ) is much greater than that for the slow branch (merely 0.3). 
Figure 2: The fast (solid lines) and slow (dashed lines) branch combustion wave solution profiles for temperature (red) and fuel concentration (blue) for $\beta=3.3, \mathrm{Le}=2, q=5, r=25, f=3$ and $l=5 \times 10^{-4}$.

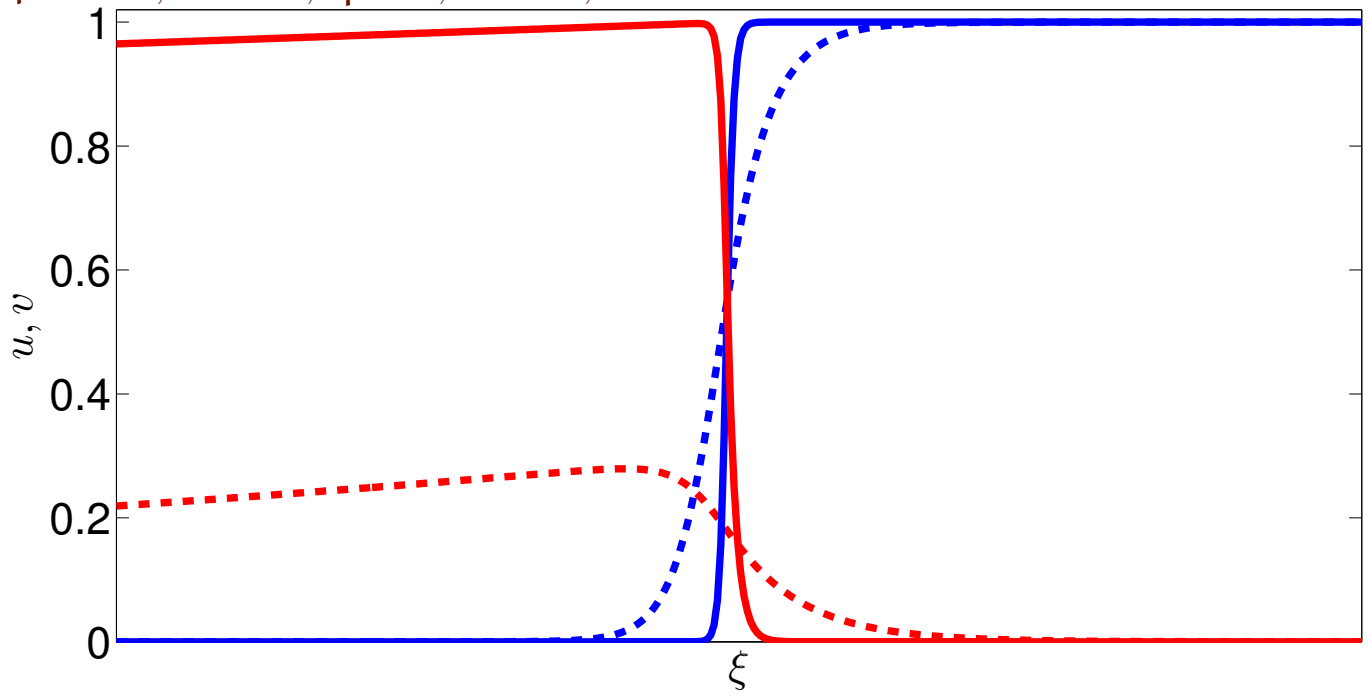

To understand the effect of the parameter $A$, we set $\beta=3.3$ and use the same three values of $l$ used in Figure 1. To ensure consistency, we employed two independent numerical approaches-MOL and FlexPDE ${ }^{\mathrm{TM}}$. In all of our numerical investigation we found that the absolute errors for the calculated flame speed are less than $\mathbf{0 . 0 0 1}$. As shown in Table 1, not all initial conditions evolve into travelling flame fronts: there exist threshold values of $A$.

Figure 3 summarises the different types of solutions accessible in the $(\beta, l)$ parameter space. We only consider the narrow interval of $3.00 \leqslant \beta \leqslant 3.42$ as the regions of bi-stability exist in this interval for some values of $l$ (recall that for $l=10^{-3}$ (magenta triangle curve in Figure 1), there is only one unique solution branch). There are three loci-the boundaries of extinction of combustion waves (red), and the onset (blue) and extinction (green) of the bi-stability. Only if the heat loss coefficient is between the green and blue curves may the fast and slow branches co-exist, that is, $l<9.4 \times 10^{-4}$. 
Table 1: Summary of results when the amplitude of the initial conditions is given by equation (9) for the three cases $l=0, l=10^{-4}$ and $l=5 \times 10^{-4}$ with $\beta=3.3$.

\begin{tabular}{|l|r|l|}
\hline Cases & Amplitude of Gaussian & Solution behaviour \\
\hline \multirow{3}{*}{$\mathrm{l} 0$} & $0 \leqslant A \leqslant 0.182$ & No combustion wave \\
& $0.182<A<0.23$ & Slow branch with $c \approx 0.152$ \\
& $0.23 \leqslant A$ & Fast branch with $c \approx 1.04$ \\
\hline $\mathrm{l}=1 \times 10^{-4}$ & $0 \leqslant A \leqslant 0.182$ & No combustion wave \\
& $0.182<A<0.23$ & Slow branch with $c \approx 0.146$ \\
& $0.23 \leqslant A$ & Fast branch with $c \approx 1.0395$ \\
$l=5 \times 10^{-4}$ & $0 \leqslant A \leqslant 0.184$ & No combustion wave \\
& $0.184<A<0.22$ & Slow branch with $c \approx 0.116$ \\
& $0.22 \leqslant A$ & Fast branch with $c \approx 1.034$ \\
\hline
\end{tabular}

Following Huang et al. [13], we investigate the location of the extinction boundary as an implicit function of $l$ and $\beta$. For instance, we find that no combustion wave is possible for $l \geqslant 9 \times 10^{-3}$ when $\beta=3.3$ but the flame speed is around 0.809 when $l=8.9 \times 10^{-3}$. Thus $l=9 \times 10^{-3}$ and $\beta=3.3$ represents a point on the extinction boundary. As $\beta$ increases extinction occurs for decreasing values of $l$. This is physically reasonable. According to the rate equation (6), $\beta$ is the coefficient of the the reaction rates. Thus, increasing $\beta$ implies the reactant is consumed faster, the reactions complete more quickly and less energy is generated to sustain the propagation of the combustion wave. Therefore, when the heat loss coefficient is large, $\beta$ should be sufficiently small to form a propagating combustion wave.

Finally, we demonstrate the possibility of switching between the fast and slow branches. Towers et al. [11] illustrated that the fast (slow) reaction could switch rapidly to the slow (fast) reaction: displaying 'jump' and 'drop' transitions between the branches by 'pushing' the value of $\beta$ slightly past the hysteresis region. Instead of perturbing $\beta$ (which can be done experimentally by 'doping' the fuel sample), we propose an alternative mechanism based on 
Figure 3: Different wave solutions in the $(\beta, l)$ parameter space for $L e=2$, $q=5, r=25$ and $f=3$. The red curve represents the extinction boundary. The blue and green curves represent the onset and extinction boundaries for the bi-stability region. NCW represents the region where no combustion waves exist, UCW denotes the region with unique combustion wave and BSR corresponds to the region where bi-stability occurs.

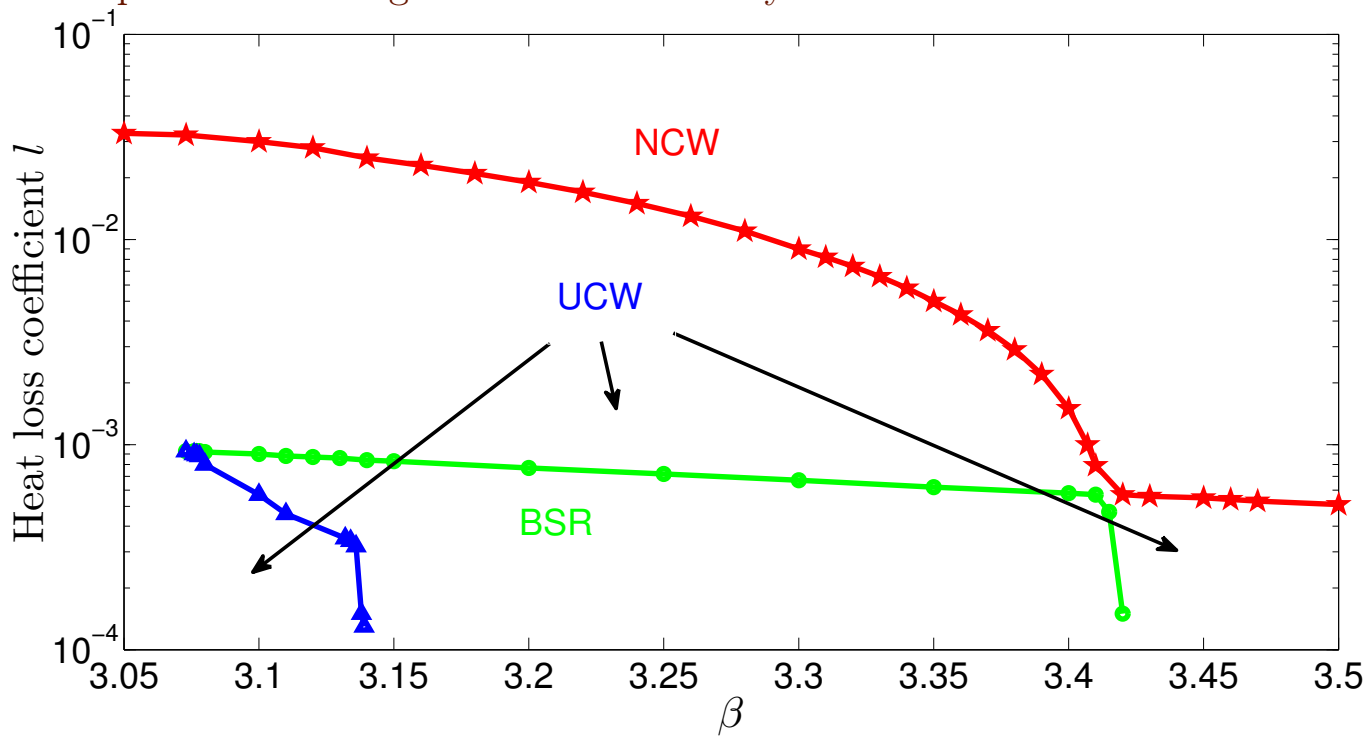

changing the temperature profile. This is physically possible and reasonable as we change the flame temperature by increasing or decreasing the ambient temperature.

We present two numerical simulations to illustrate the transitions between the fast and slow branches. For each case, once the temperature and fuel profiles were found for $\beta=3.3, \mathrm{Le}=2, \mathrm{q}=5, \mathrm{r}=25, \mathrm{f}=3$ and $l=5 \times 10^{-4}$, the temperature profile was then varied. Using these altered profiles as the initial conditions for the governing PDEs, the observed transitions are shown in Figures 4 and 5 . These solutions are achieved by initially integrating the PDEs over the time domain $0 \leqslant \tau \leqslant 1500$ so that a stable flame front is formed 
Figure 4: The behaviour of the flame front speed as the temperature profile is increased by $10 \%, 50 \%$ and $100 \%$ (curves labelled 1.1, 1.5 and 2, respectively) at $\tau=1500$ for $\beta=3.3$, Le $=2, q=5, r=25, f=3$ and $l=5 \times 10^{-4}$. The solutions on the slow branch have speed $\mathrm{c} \approx 0.116$, whereas the solutions on the fast branch have speed $c \approx 1.034$.

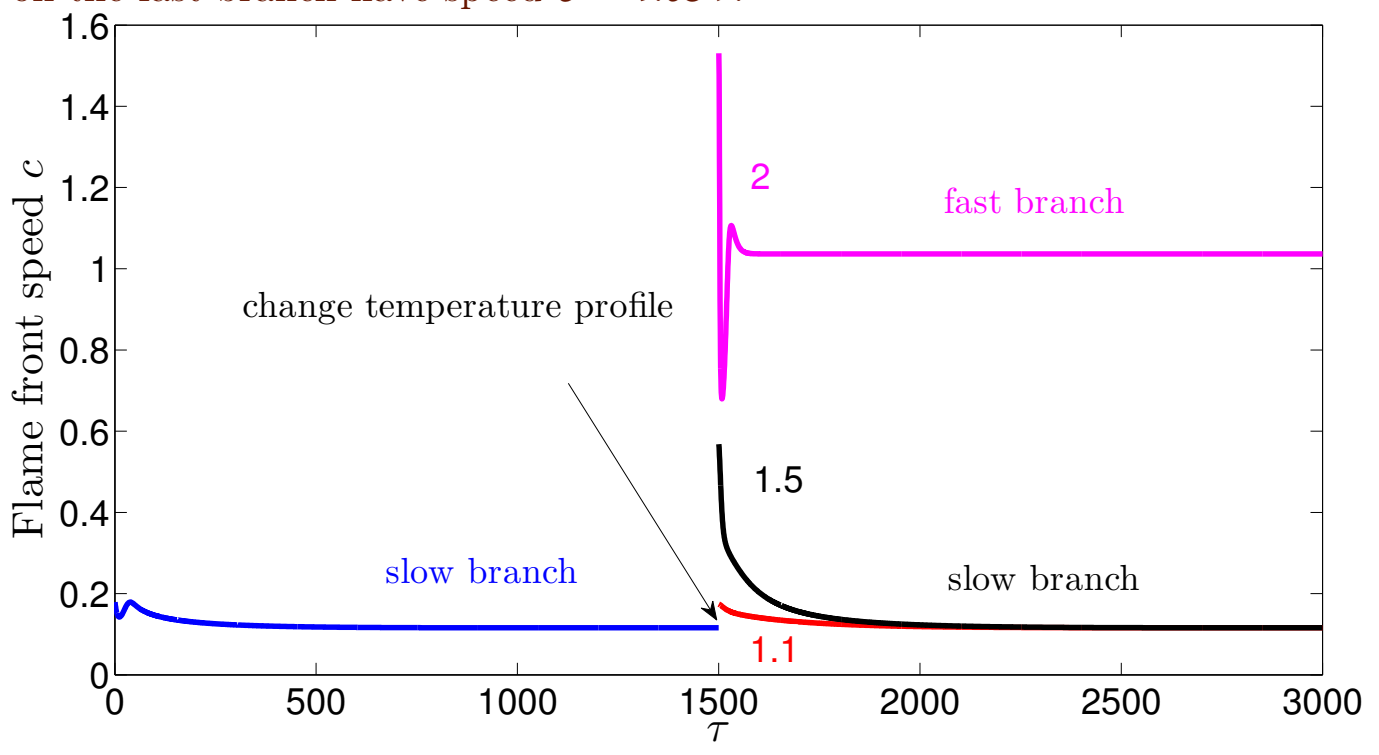

which propagates at a constant flame speed. Then the system is integrated from $\tau=1500$ with the 'perturbed' temperature profile, while the fuel profile remains unchanged. The flame front speed undergoes a transitory phase in the interval $1500 \leqslant \tau \leqslant 1800$ to reach a steady state value. Figure 4 shows the propagation of a flame front initially converging on the slow branch that is perturbed at $\tau=1500$. When we increase the temperature profile by $10 \%$, after an initial transition, the solution returns to the slow branch. Similar behaviour is observed when the temperature profile was increased by $50 \%$. However, a 100\% increase in the temperature profile results in the solution converging onto the fast branch. The 'jump up' transition occurs over a small time interval of about 100 time units. 
Figure 5: The behaviour of the flame front speed as the temperature profile is decreased by $10 \%, 50 \%$ and $90 \%$ (curves labelled 0.9, 0.5 and 0.1 , respectively) at $\tau=1500$ for the same parameter values as those in Figure 4 . The solutions on the fast branch have speed $c \approx 1.034$, whereas the solutions on the slow branch have speed $c \approx 0.116$.

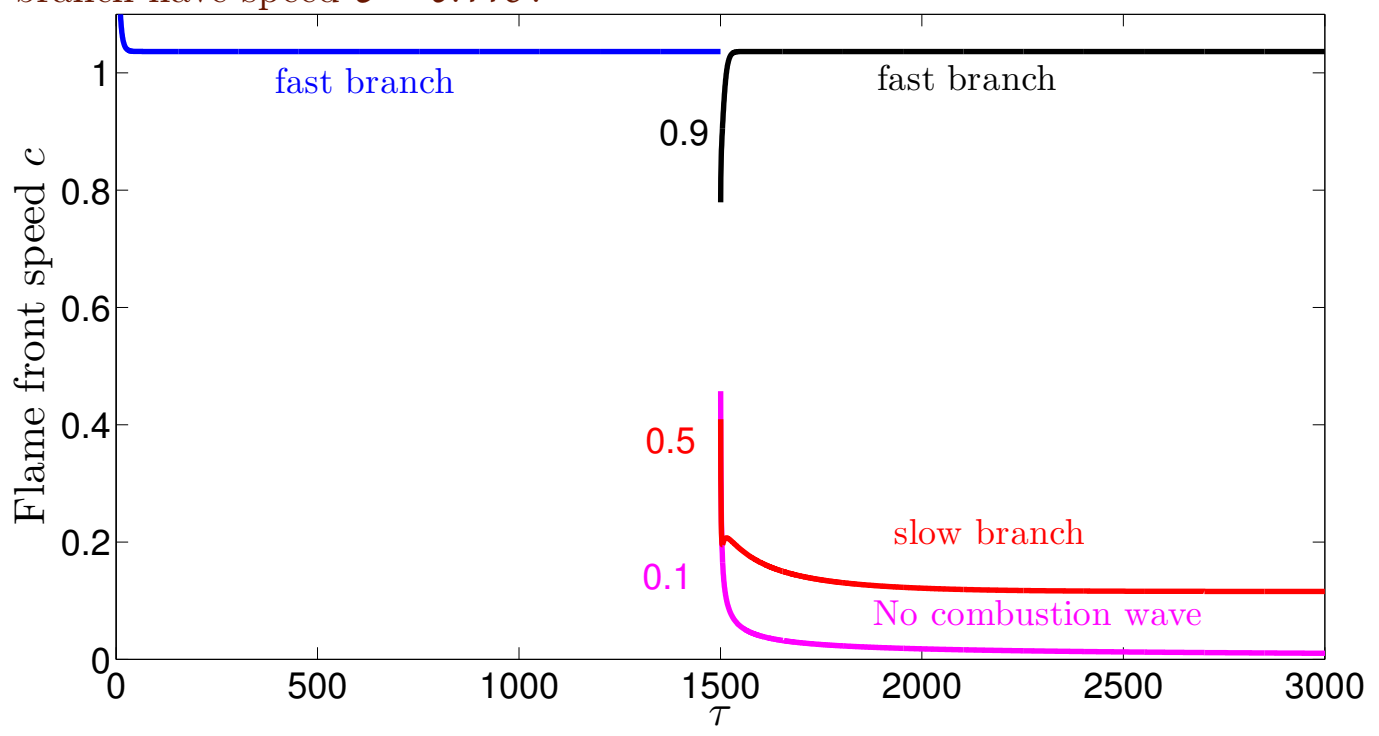

Figure 5 shows the 'drop down' effect from the fast to the slow branch when the temperature profile is reduced at $\tau=1500$. We see that a $10 \%$ reduction results in the solution converging back onto the fast branch. However, a 50\% reduction results in the solution moving away from the fast branch and settling on the slow branch. Moreover, a massive $90 \%$ reduction in the amplitude of the temperature profile ensures that no combustion wave is sustainable.

By undertaking the same perturbed temperature process for the adiabatic case, we find that there is only a minor effect in the perturbation for a 'drop down' switch to occur when compared to the nonadiabatic case (a difference of only around $1 \%$ in the temperature decrease between the two cases). However, the inclusion of heat loss has a greater impact in the 'jump up' switch (a 
difference of $16 \%$ in the temperature increase between the adiabatic and nonadiabatic cases). Hence, including heat loss into the system has some 'stabilizing' effect within the bi-stability region.

\section{Conclusion}

We presented a preliminary investigation of a one-dimensional reactiondiffusion system as a model for the propagation of combustion waves with a two-step competitive exothermic-reaction and heat loss. Numerical solutions of the governing PDEs were obtained using FlexPDE ${ }^{\mathrm{TM}}$ and confirmed using MOL. We demonstrated that the flame front speed decreases as the heat loss coefficient increases, since increasing the heat loss means losing more energy to the surroundings, thus making less energy available to form and sustain the flame fronts. When there is too much heat loss, the flame front cannot propagate. Our earlier work in the adiabatic case showed the existence of regions of bi-stability - coexistence of stable solutions corresponding to the fast and slow branches $[10,11]$. Such multi-stable solutions are also observed experimentally (e.g., Hall and Wolfhard [18] showed the existence of three types of ethyl nitrate-air propagating flames). In this article we showed that bi-stability can still exist for the nonadiabatic case, but only if the heat loss parameter is below some threshold value. The combustion waves can be switched from one branch to the other by perturbing the temperature profiles. Larger perturbations are required for the 'jump up' switch to take place in the nonadiabatic case compared to the adiabatic case. However, the inclusion of heat loss appears to have very little effect in the 'drop down' transition. Nevertheless, switching can result in a rapid increase (or decrease) in the flame front speed as well as in temperature (see Figure 2), which may have serious safety implications for mines, industries and bush-fires. This switching behaviour may also be of interest in the combustion synthesis of advanced materials. 


\section{References}

[1] W. Choi, S. Hong, J. T. Abrahamson, J.-H. Han, C. Song, N. Nair, S. Baik and M. S. Strano. Chemically driven carbon-nanotube-guided thermopower waves. Nat. Mater. 9:423-429, 2010. doi:10.1038/nmat2714 C15

[2] A. G. Merzhanov. Combustion and explosion processes in physical chemistry and technology of inorganic materials. Russ. Chem. Rev. 72:289-310, 2003. doi:10.1070/rc2003v072n04abeh000766 C16

[3] J. K. Bechtold and C. K. Law. The structure of premixed methane-air flames with large activation energy. Combust. Flame 97:317-338, 1994. doi:10.1016/0010-2180(94)90024-8 C16

[4] K. Seshadri, N. Peters and F. A. Williams. Asymptotic analysis of stoichiometric and lean hydrogen-air flames. Combust. Flame 96:407-427, 1994. doi:10.1016/0010-2180(94)90108-2 C16

[5] A. L. Sánchez, A. Lépinette, M. Bollig, A. Liñán and B. Lázaro. The reduced kinetic description of lean premixed combustion. Combust. Flame 123:436-464, 2000. doi:10.1016/S0010-2180(00)00177-2 C16

[6] A. L. Sánchez, G. Balakrishnan, A. Liñán and F. A. Williams. Relationships between bifurcation and numerical analyses for ignition of hydrogen-air diffusion flames. Combust. Flame 105:569-590, 1996. doi:10.1016/0010-2180(95)00241-3 C16

[7] V. V. Gubernov, A. V. Kolobov, A. A. Polezhaev and H. S. Sidhu. Analysing the stability of premixed rich hydrogen-air flame with the use of two-step models. Combust. Flame 160:1060-1069, 2013. doi:10.1016/j.combustflame.2013.01.021 C16

[8] R. Ball, A. McIntosh and J. Brindley. Thermokinetic models for simultaneous reactions: a comparative study. Combust. Theor. Model. 3:447-468, 1999. doi:10.1088/1364-7830/3/3/302 C17 
[9] N. A. Martirosyan, S. K. Dolukhanyan and A. G. Merzhanov. Nonuniqueness of stationary states in combustion of mixtures of zirconium and soot powders in hydrogen. Combust. Explo. Shock 19:569-571, 1983. doi:10.1007/BF00750423 C17

[10] H. S. Sidhu, I. N. Towers, V. V. Gubernov, A. V. Kolobov and A. V. Polezhaev. Investigation of flame propagation in a model with competing exothermic reactions. Chemeca 2013, Brisbane, Australia, 29 September-2 October, 2013.

http://www. conference.net.au/chemeca2013/papers/29350.pdf C17, C20, C28

[11] I. N. Towers, V. V. Gubernov, A. V. Kolobov, A. V. Polezhaev and H. S. Sidhu. Bistability of flame propagation in a model with competing exothermic reactions. Proc. R. Soc. Lond. A 469:20130315, 2013. doi:10.1098/rspa.2013.0315 C17, C22, C24, C28

[12] L. K. Forbes and W. Derrick. A combustion wave of permanent form in a compressible gas. Anziam J. 43:35-58, 2001. doi:10.1017/S144618110001141X C18

[13] Z. Huang, H. S. Sidhu, I. N. Towers, Z. Jovanoski and V. V. Gubernov. Investigating flame fronts in competitive exothermic reactions. MODSIM2015, 21st International congress on Modelling and simulation, Gold coast, Australia, 29 November-4 December, 2015. http://www.mssanz.org.au/modsim2015/A1/huang.pdf C18, C24

[14] F. A. Williams. Combustion theory. Westview Press, 1985. https://westviewpress. com/?s=Combustion+theory C19

[15] FlexPDE ${ }^{\mathrm{TM}}$, PDE Solutions Inc. http://www.pdesolutions.com C20

[16] W. E. Schiesser. The numerical method of lines: integration of partial differential equations. Academic Press, San Diego, 1991. http://store.elsevier.com/The-Numerical-Method-of-Lines/ isbn-9780128015513/C20 
[17] A. C. McIntosh, R. O. Weber and G. N. Mercer. Non-adiabatic combustion waves for general Lewis numbers: wave speed and extinction conditions. Anziam J. 46:1-16, 2004. doi:10.1017/S1446181100013638 $\mathrm{C} 22$

[18] A. R. Hall and H. G. Wolfhard. Multiple reaction zones in low pressure flames with ethyl and methyl nitrate, methyl nitrite and nitromethane. Proc. Combust. Inst, 6:190-199, 1957. doi:10.1016/S0082-0784(57)80029-0 C28

\section{Author addresses}

1. Z. Huang, Applied and Industrial Mathematics Research Group, School of Physical, Environmental and Mathematical Sciences, UNSW, Canberra, Australia.

mailto: zhe . huang@student. adfa.edu.au

2. H. S. Sidhu, Applied and Industrial Mathematics Research Group, School of Physical, Environmental and Mathematical Sciences, UNSW, Canberra, Australia.

3. I. N. Towers, Applied and Industrial Mathematics Research Group, School of Physical, Environmental and Mathematical Sciences, UNSW, Canberra, Australia.

4. Z. Jovanoski, Applied and Industrial Mathematics Research Group, School of Physical, Environmental and Mathematical Sciences, UNSW, Canberra, Australia.

5. V. V. Gubernov, I.E. Tamm Theory Department, P.N. Lebedev Physical Institute, 53 Leninsky Prospect, 119991 Moscow, Russia. 